Pensamiento Crítico № 16, pp. 37-49

\title{
La paradoja: crecimiento y desarrollo economico en el Perú (2001 - 2010)
}

Mg. Víctor Orozco Livia

\section{RESUMEN}

Una paradoja es una contradicción, que según Ferrater Mora en su Diccionario de Filosofía Abreviado, debía interpretarse etimológicamente como contrario a la opinión recibida y común.

Para el caso del tema tratado en este trabajo seria la opinión difundida por las autoridades responsables de la política económica en el Perú, pero que en los hechos resulta contraria o paradójica a lo que se afirma.

En el Diccionario Filosófico de Rosental y ludin se agrega que una paradoja plantea el problema de hallar la fuente de tal contrariedad y los procedimientos para eliminarla.

Por consiguiente, el trabajo de investigación titulado "La Paradoja: Crecimiento y Desarrollo Económico en el Perú", implica conocer en primer lugar cuales son las causas de esta contradicción económica, para luego en un segundo momento proponer medidas económicas que tiendan a resolverla.

Las causas que dan lugar a la paradoja económica en el Perú son varias, abarca aspectos económicos, sociales, políticos, tecnológicos, filosóficos, epistemológicos, ideológicos, etc., que por cierto no serán abordadas en su totalidad en este trabajo, pero que si serán asumidas cuando la ocasión lo exija.

Palabras clave: Paradoja-Crecimiento-Desarrollo-Globalización-Neoliberalismo. 


\title{
Pensamiento Crítico $\mathbf{N}^{\circ}$ I6
}

\begin{abstract}
A paradox is a contradiction which according Ferrater Mora in his Dictionary of Fhilosophy Short, etymologically be interpreted as contrary to received opinion and common.

In the case of the subject of this work would be the opinion released by the authorities responsible for economic policy in Peru, but which in fact is contrary or paradoxical to what is stated.

In the Philosophical Dictionary Iudin Rosental and adds that a paradox arises the problem of finding the source of such annoyance and procedures to eliminate it.

Therefore, the paper titled "The Paradox: Growth and Economic Development in Peru", means knowing first what are the causes of this economic contraction, then a second time propose economic measures that tend to resolve it.

The causes that lead to economic paradox in Peru are varied, covering economic, social, political, technological, philosophical, epistemological, ideological, etc., which certainly will not be fully addressed in this work, but if be assumed when the occasion demands.
\end{abstract}

Keywords: Paradox, growth, development, globalization, neoliberalism.

\section{La nocion de crecimiento y desarrollo economico}

\section{Crecimiento y Desarrollo Económico}

Crecimiento y Desarrollo Económico no son sinónimos aunque algunos economistas utilizan indistintamente estos conceptos considerando que se refieren a lo mismo. El crecimiento es el aumento progresivo de la cantidad de bienes y servicios producidos en la economía, en un período determinado. El desarrollo económico de un país se refleja cuando aumenta la producción pero también la calidad de vida de la población en general.

El crecimiento económico como único propósito de política económica puede generar una grave desigualdad entre la población, porque propugnaría básicamente un aumento de utilidades, que es lo que en última instancia significa el aumento del Producto Bruto Interno. Por un lado los que obtienen utilidades estarían muy bien y los 


\section{Víctor Orozco Livia}

que reciben remuneraciones irrisorias por su trabajo estarían muy descontentos. Los primeros son minorías y los segundos, mayoría. Son desigualdades que pueden ser reducidas si los gobiernos, en la eventualidad de que el crecimiento sea continuo, podrían implementar políticas de redistribución de ingresos y generación de fuentes de trabajo productivo, tanto en el sector público como en el privado. Con ello se incorporaría a una mayor cantidad de personas al aparato productivo. Con un crecimiento económico sostenido y una política económica no sesgada a la minoría, podría lograrse mayores niveles de bienestar a la población, que es el objetivo del Desarrollo Económico.

\section{Los Efectos Sociales de la Desigualdad}

En un artículo que publica el sociólogo Pedro Francke $e^{1}$ en un diario nacional referido a los efectos sociales que genera la desigualdad en una sociedad considera los siguientes:

- La gente vive menos y la esperanza de vida es menor

- Su gente es menos feliz

- Crecen económicamente menos

- La confianza entre sus ciudadanos es menor

- El status social de las mujeres es más bajo

- Hay más personas mentalmente enfermas

- Consumen más drogas

- La movilidad social es menor

- Hay más adultos y niños obesos

- Los niños aprenden menos y tienen menos logros educativos

- Hay más embarazos adolescentes y más abortos

- La tasa de homicidios es mayor

- Los niños son más propensos a la violencia

- Hay más presos en las cárceles

1 Francke, Pedro. Diaria La Primera Martes, 28 de Diciembre de 2010. Pag, 10 


\section{Pensamiento Crítico $\mathbf{N}^{\circ} 16$}

De acuerdo a las implicancias sociales que encuentra este autor por causa de la desigualdad en una sociedad, resulta que el problema no es estrictamente económico sino también de carácter político, económico, social, etc., que agobia a un país en el que existe una paradoja resaltante entre Crecimiento Económico y Desarrollo Económico.

Una política económica neoliberal implementada por políticos representantes de inversionistas nacionales, poco conscientes de sus valores nacionales, emparentados con inversionistas extranjeros apoyados por sus respectivos gobiernos, para los cuales lo único que interesa es la maximización de la tasa de ganancia, puede generar desequilibrios muy pronunciados entre los miembros de una sociedad como la nuestra.

\section{El contexto nacional e internacional}

\section{La permisibilidad del neoliberalismo en el perú}

La implementación del neoliberalismo en el Perú encontró determinadas condiciones internas favorables que dieron lugar a su aplicación.

En primer lugar está el ineficiente resultado de la gestión del Primer Gobierno del Partido Aprista Peruano. Sólo para mencionar dos aspectos de esta administración, está por un lado la hiperinflación y por otro lado la suspensión del pago de la Deuda Externa, cuyos significados fueron la minimización del salario real que empobreció a la población mayoritaria, y por otra parte la morosidad en la Deuda Externa que calificó al Perú como país no elegible de créditos internacionales. El viraje exigido por empresarios nacionales y extranjeros ante estos hechos, dio lugar a ciertas condiciones para volver a políticas económicas tradicionales.

En segundo lugar, están los valores económicos, sociales, políticos, éticos, ideológicos, etc., que dejaron como herencia los terratenientes de la época colonial y mantenidos en gran parte de la vida republicana. Con una breve excepción en el período 1968-1975, cuando un gobierno reformista militar, encabezado por Juan Velasco Alvarado pretendió reformular estos valores. En este gobierno se hicieron grandes reformas económicas, cuyo mayor logro fue la Reforma Agraria, que desplazó del poder económico y político a los terratenientes, pero que en última instancia quedó trunca y frustrada la posibilidad de extinguir tales valores tradicionales. 


\section{Víctor Orozco Livia}

Producida la caída de Velasco Alvarado se mantuvo por inercia aquellos valores inculcados por los terratenientes, de tal manera que los gobernantes que sucedieron a Velasco y controlaron nuevamente el Ministerio de Educación retornaron a difundir y mantener esos valores que sirvieron para acoger al Neoliberalismo en el Perú.

Desde el punto de vista político los terratenientes siempre consideraron al capital Norteamericano y su gobierno como aliados naturales en sus quehaceres políticos, y así quedó impregnado en la conciencia política de las clases dominantes en el Perú. Aún a mediados de la década del 90 se presentaba como nueva teoría la creencia de que "el mundo del presente es un mundo interdependiente; ahora no solo los países en desarrollo dependen de los países desarrollados, sino que también estos últimos tienen que medir sus actos en función a las acciones de los países en desarrollo" 2

En el escenario social los terratenientes en el poder dejaron también como herencia a las clases dominantes que les sucedieron, valores laborales de explotación y marginación a los trabajadores, ya que por mucho tiempo solo les interesó la renta de la tierra, la expansión de sus predios rurales, el pago en especies a los trabajadores rurales, marginación a la educación y escaso acceso a cargos públicos importantes. Asimismo, no permitieron el desarrollo industrial del Perú y con ello se opusieron a la modernización del país. Facilitaron la inversión extranjera y no se preocuparon por el desarrollo del capital industrial nacional.

En lo que respecta los valores éticos que conserva la clase dominante en el Perú, sólo haría falta hacer referencia a las tendencias de corrupción, heredadas desde los tiempos coloniales, que se ha convertido hoy en día en un mal endémico, impregnado y expandido como en el Sector Público en el Perú. El Neoliberalismo se sirvió de esos valores éticos en la clase dominante del Perú para facilitar acuerdos ilegales entre el gobierno y los grandes inversionistas extranjeros y nacionales, tal y como quedara grabado en videos y audios, como los que aparecieron durante el gobierno de Fujimori y García Pérez.

2 Morales, Raymundo. Del aislamiento a la reinserción I.de E. de L.M. Lima 1995 


\section{Pensamiento Crítico $\mathbf{N}^{\circ} 16$}

\section{La Primera Experiencia Monetarista en Latinoamérica}

El Monetarismo, base del Neoliberalismo, se difundió desde la década del 70, como preocupación y respuesta del gobierno norteamericano a los llamados "gobiernos populistas", que aparecieron en el Perú con Juan Velasco Alvarado, en Bolivia con Juan José Torres y en Chile con Salvador Allende.

La Conspiración y derrocamiento del gobierno de Salvador Allende en Chile, comandada por la alianza entre la clase dominante chilena y los intereses norteamericanos, fue la oportunidad para el ensayo experimental del Monetarismo en Latinoamérica.

El gobierno de Pinochet y los "Chicago Boys", discípulos de Milton Friedman que arribaron a Chile impusieron bajo tal dictadura una política monetarista, constituida entonces como instrumento político del Gobierno Norteamericano. Los resultados no fueron precisamente halagadores, porque los sucesivos gobiernos en Chile de Alwin, Lagos y Bachelet, constituyeron expresiones de rechazo al Monetarismo.

\section{El Neoliberalismo en el Perú}

El fracaso de la política económica del gobierno del Partido Aprista en el Perú en el período 1985-1990, considerado "populista" y no amigo por el gobierno norteamericano, y las concepciones derechistas de las clases dominantes en el Perú, propugnaron por un retorno a las posiciones políticas tradicionales.

Principalmente la hiperinflación que afectó al ingreso real de muchos peruanos y la declaración de país inelegible para créditos externos por parte de organismos como el Fondo Monetario Internacional y el Banco Mundial, más la suspensión del pago de la deuda externa dieron lugar a que el electorado eligiera a un candidato prácticamente desconocido como lo era en aquel entonces el Ing. Alberto Fujimori como Presidente de la República en 1990.

El Programa Económico que esgrimió inicialmente el candidato Alberto Fujimori fue el de llevar a cabo reformas progresivas, para estabilizar la economía. 


\section{Víctor Orozco Livia}

En estos afanes el equipo de asesores en asuntos económicos del Ing. Fujimori lo lideraba el prestigioso economista Adolfo Figueroa, profesor de la Pontificia Universidad Católica del Perú.

Cuando ganó las elecciones el Ing. Fujimori viajó a Estados Unidos, se entrevistó con funcionarios del gobierno norteamericano, del FMI y el BM, y cuando retornó al Perú despidió a su equipo de asesores y nombró como Ministro de Economía a un Ingeniero Agrónomo, Juan Hurtado Miller. ¿Qué había pasado?

En el interin de esas conversaciones con representantes norteamericanos, éstos convencieron al Presidente electo de que la única forma de resolver los graves problemas de la economía peruana era la sujeción al "Consenso de Washington". De este modo Perú tendría el apoyo de esas instituciones financieras para reinsertarse en el mundo financiero internacional. Por este acuerdo no se requería de un economista como Ministro de Economía, sino tan sólo un amigo de la Universidad Agraria La Molina, ya que desde entonces el gobierno confió en las recomendaciones del FMI para lograr la estabilización de la economía peruana.

Se aplicó una política de shock recomendada por el FMI, siguiendo los lineamientos de política económica derivados de la teoría monetarista y del "Consenso de Washington". La fuerte oposición expresada por la población y el Congreso dio lugar a un golpe de Estado. El Presidente Fujimori disolvió el Congreso en 1992 y empezó a gobernar en forma dictatorial dejando las condiciones expeditas para la aplicación del Neoliberalismo en el Perú.

\section{Los efectos del neoliberalismo en el peru}

\section{Aplicaciones y Resultados del Neoliberalismo}

A partir del 5 de Abril de 1992, con el cierre del Congreso y el inicio del Gobierno Dictatorial que presidió el Ing. Alberto Fujimori, el Neoliberalismo se hizo presente en el Perú por acuerdos entre el Gobierno Peruano y el Gobierno Norteamericano. 


\section{Pensamiento Crítico $\mathrm{N}^{\circ} 16$}

Desde entonces hasta el 2011 que se produjo el cambio a un Gobierno Nacionalista, estuvo vigente el Neoliberalismo con los gobiernos de Fujimori, Toledo y García.

La política económica neoliberal aplicada en el Perú ha generado una desigualdad muy acentuada entre los que se benefician con el incremento del PBI y la mayoría de la población peruana que no ha experimentado una elevación de bienestar en su existencia.

\section{i. El Comportamiento del PBI}

El crecimiento económico de un país se expresa fundamentalmente en el incremento del PBI.

En el Perú el Producto Bruto Interno ha observado un crecimiento persistente en la última década, desde 1991 hasta 2010, en el que se registra un crecimiento promedio del PBI del 6\% anual, tal como se aprecia en el cuadro adjunto. Lo cual constituye un referente muy importante para aplicaciones de políticas económicas de redistribución de ingresos. Como esto no se llevó a cabo en estos últimos diez años, el crecimiento de bienes y servicios se ha traducido mayormente en grandes ganancias, que ha beneficiado básicamente a los empresarios de los bienes de capital y empresarios de los bienes y servicios ofertados al mercado. Los productores directos con el despliegue de sus energías físicas y mentales, y como creadores de los bienes y servicios, no han percibido los resultados de esta espiral ascendente de producción y servicios, reflejándose en sus bajos ingresos y congelación de la calidad de vida.

El "Consenso de Washington" aplicado en el Perú generó el crecimiento económico por diversas vías. La facilitación de inversiones extranjeras, la apertura del mercado interno a las mercancías de los conglomerados multinacionales que ocasionaron la quiebra de alrededor de 300 mil empresas nacionales, el recorte de los derechos laborales para propiciar mayores ganancias, etc.

El aumento continuo de la producción reflejó una política económica sesgada a las grandes inversiones extranjeras y nacionales, y escaso interés por el estado de los trabajadores pertenecientes a las grandes mayorías nacionales. 


\section{Víctor Orozco Livia}

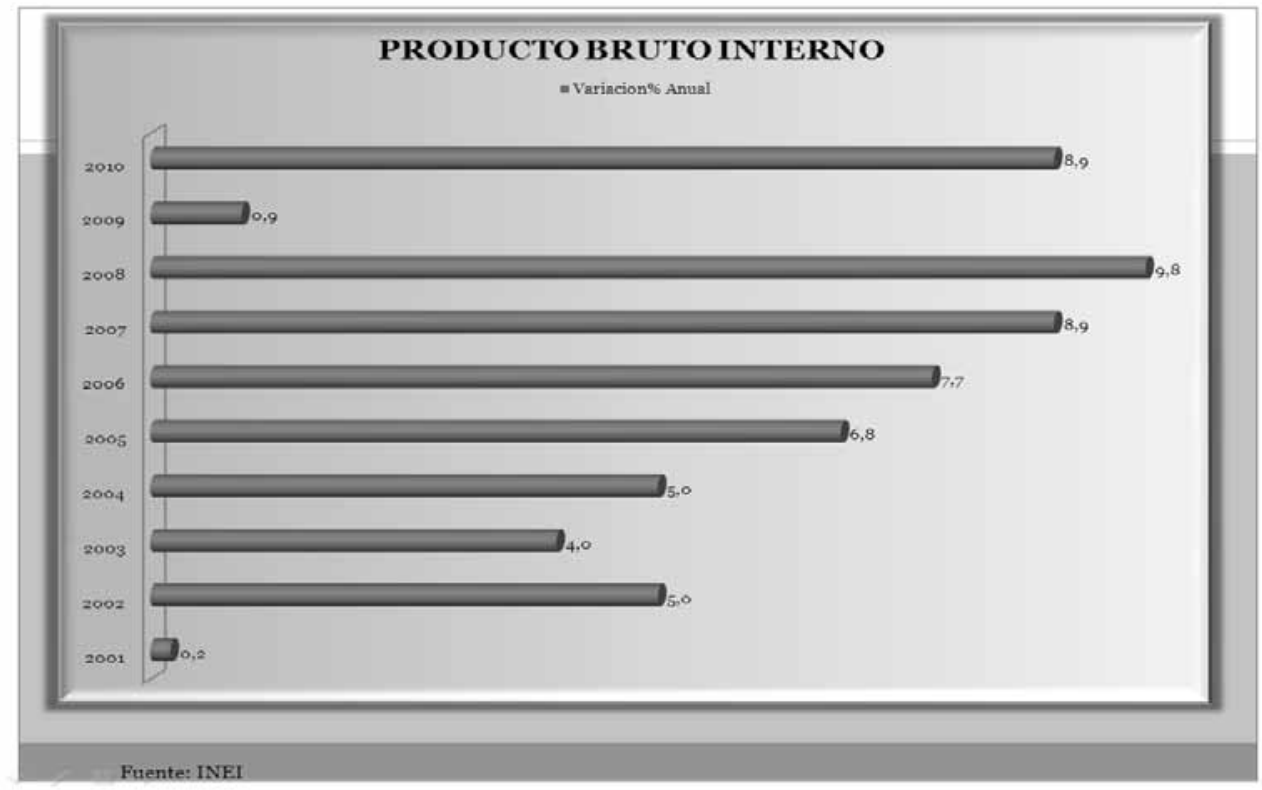

\section{ii. Remuneración Mínima Vital}

El salario mínimo vital refleja la apreciación que tienen los gobernantes respecto a la calidad de vida que se ofrece a las grandes mayorías de trabajadores de un país.

En el Perú, cuyos últimos tres gobernantes del país, se guiaron por las recomendaciones del "Consenso de Washington", mantuvieron un salario mínimo vital que fue uno de los bajos de la región, alcanzando a Diciembre de 2010 a la cifra de US $\$ 211$. Comparado con otros países tenemos que en Chile es de US \$333, en Ecuador US \$340, en Colombia US \$286, en Argentina US \$379 y solo superamos a Bolivia que se fijó en US \$97.05

Lo sorprendente en la política económica neoliberal en el Perú es que mientras las ganancias aumentaban progresivamente, el salario mínimo vital se mantenía como uno de los más bajos en la región tal y como puede notarse en el siguiente cuadro que se refiere a Diciembre 2010. 


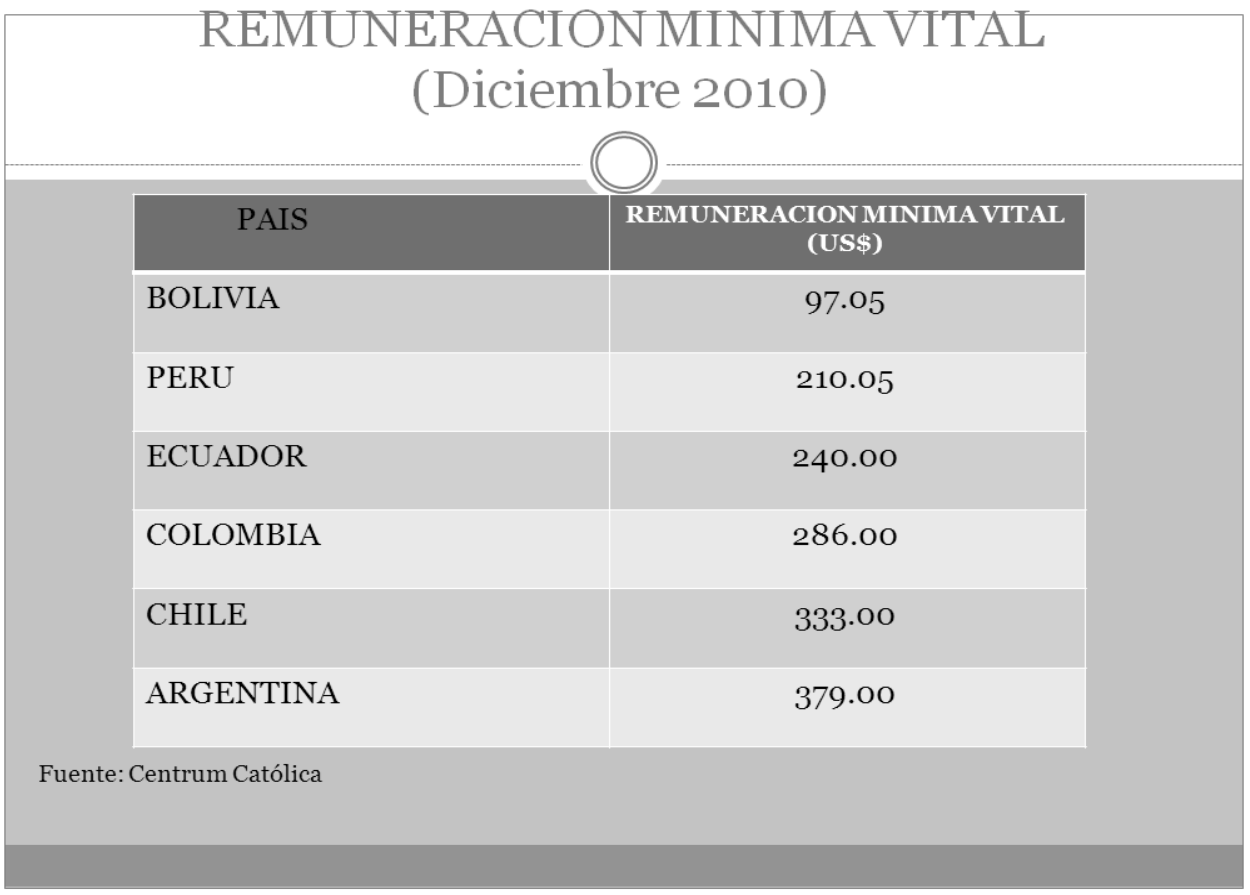

\section{iii. Participación porcentual en el PBI del Excedente de Explotación y las Remuneraciones}

La relación entre ganancias y remuneraciones como porcentajes del PBI se ha distanciado en la última década como resultado de aplicaciones de políticas neoliberales. Especialmente las disposiciones en el Art. 27 de la Constitución de 1993 por las que el trabajador pierde el derecho de estabilidad laboral y se permite el despido del trabajador por cualquier causa, posibilitaron el congelamiento del salario mínimo vital, que hasta Diciembre del 2011 solo alcanzaba a 580 nuevos soles, el segundo más bajo de la región, ligeramente superior al de Bolivia que estaba en el último escalón.

Con ello las inversiones, sobre todo extranjeras de los conglomerados multinacionales, pudieron obtener elevadas ganancias, de tal modo que la brecha entre ganancias y remuneraciones como porcentajes del PBI, fue creciendo tal y como se puede apreciar en el cuadro que a continuación se presenta. 


\section{Víctor Orozco Livia}

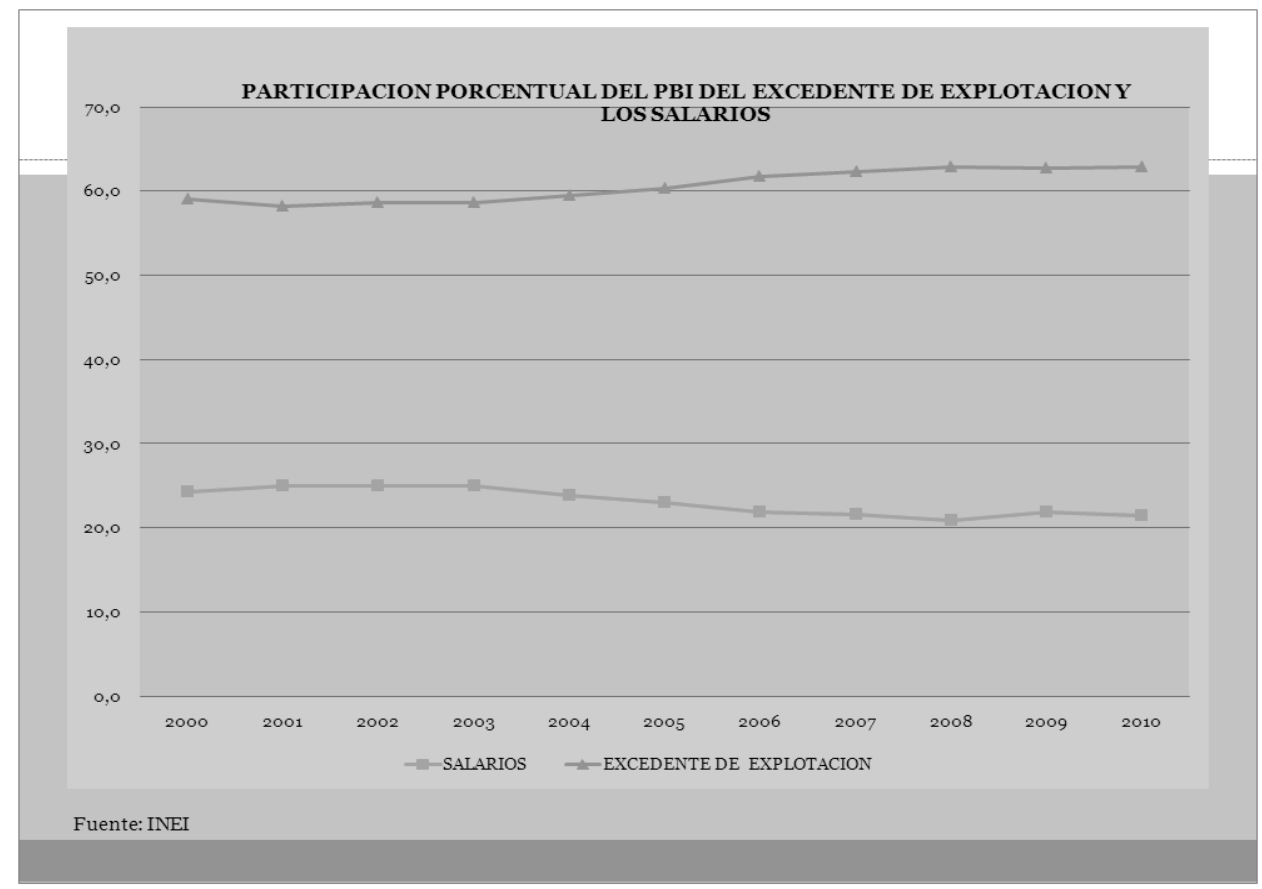

\section{UN MODELO NACIONALISTA CON REDISTRIBUCION DE INGRESOS}

Si el Neoliberalismo en el Perú, significa básicamente una gran inversión extranjera y escasa inversión de capitales nacionales, con ganancias muy altas y bajos salarios, se requiere de un modelo que revierta esta situación.

En primer lugar se requiere de un gobierno que priorice el desarrollo del capital nacional, de tal modo que las reinversiones por utilidades tiendan a forjar un mercado nacional y no un mercado para el capital extranjero. En segundo lugar, si las utilidades que obtienen los inversionistas extranjeros y nacionales en el Perú son muy elevadas, existen condiciones para llevar a cabo una política económica de redistribución de ingresos para elevar la calidad de vida de la población peruana.

A corto plazo se requiere de parte del gobierno una mayor exigencia a los inversionistas a cumplir con sus obligaciones, sobre todo a las concesiones de industrias extractivas. Muchas de ellas no cumplen con sus obligaciones tributarias. 


\section{Pensamiento Crítico $\mathrm{N}^{\circ} 16$}

Si no cumplen, tales concesiones deben revertir al Estado y ser otorgadas al capital nacional. Con ello se logra dos efectos, mayores recursos para las arcas fiscales y aumento del capital nacional. El desarrollo del capital nacional debe ser prioritario y la inversión extranjera complementaria.

Si con el incremento de las exportaciones, el Estado goza de buena salud financiera, con un mayor celo en el cobro de impuestos a las inversiones extranjeras estos fondos públicos crecerían más. Con ello podría llevarse a cabo una redistribución de ingresos y mayor participación del Estado en la economía. Una mayor participación del capital nacional y del Estado en la economía, daría lugar a una mayor autonomía de nuestra economía.

La política económica no sería entonces asistencialista como lo propugna el Neoliberalismo, sino de un reajuste de salarios e inversiones públicas infraestructurales y creación de empresas públicas estratégicas. Petroperú es un buen ejemplo de que con metodologías gerenciales modernas, cualquier empresa pública, puede ser competitiva y generar importantes utilidades para el Estado.

El fomento a una mayor acumulación de capital nacional, aumento de salarios, participación del Estado creando fuentes de trabajo, con inversiones públicas y estratégicas, daría lugar a un mercado nacional amplio, sólido y competitivo, con operatividad exitosa incluso en el mercado internacional. Sus efectos redundarían en un menor desempleo, menor pobreza, disminución de la tasa de mortalidad infantil, mayor bienestar a la niñez en alimentación, salud y educación, disminución de las enfermedades sociales y contrarrestación a los efectos sociales nocivos generados por el Neoliberalismo y señalados por el sociólogo Pedro Francke.

\section{Conclusiones}

1. La asimetría crecimiento y desarrollo económico obedece a una mentalidad no nacional y excluyente por parte de los gobernantes del país que ha acentuado la desigualdad.

2. La aplicación del neoliberalismo en el Perú se hizo en condiciones de un gobierno dictatorial en 1992

3. La desigualdad ha generado una serie efectos nocivos en el campo social. 


\section{Víctor Orozco Livia}

4. El Neoliberalismo se basa en el Monetarismo, que no ha resuelto los problemas de desarrollo económico en países como en Perú.

5. El instrumento político del Neoliberalismo es el Consenso de Washington, favorable a los conglomerados multinacionales y desfavorables para las mayorías nacionales.

\section{Recomendaciones}

1. Es necesario forjar una conciencia nacional, democrática e incluyente, para impulsar el desarrollo del país.

2. No se debe permitir gobiernos dictatoriales en el Perú.

3. Un modelo nacionalista con redistribución de ingresos disminuiría la desigualdad y elevaría la calidad de vida de las mayorías nacionales.

4. En materias económicas se deben hacer estudios comparativos y críticos que propugnen hallazgos de las propiedades inherentes a la economía del país.

5. Las políticas económicas en el Perú no deben ser asumidas como recomendaciones inefables cuando corresponden a otras realidades económicas.

\section{Bibliografia}

Friedman, M.Bases para un Desarrollo Económico. C. de Ed. Pop. Stgo. de Chile, 1975

Stiglitz, J. Malestar en Globalización. Ed. Taurus. Madrid, 2002

Solis, Ciro Inicio de Epistemología. Ed. San Marcos. Lima, 2007

Zurawicki, S. Problemas Metodológicos de las Ciencias Económicas. Ed. Nuestro Tiempo. México, 1972

Santisteban L. Ensayo sobre Metodología de las Ciencias Sociales. Ed. CIESUL. Lima, 1982

Morales, R. Del Aislamiento a la Reinserción. Ed. I.de Eco. de L.M. Lima, 1993 